\title{
Artigo original \\ Desenvolvimento motor e crescimento somático de crianças com diferentes contextos no ensino infantil
}

\author{
Décio Rodrigues \\ Eric Leal Avigo \\ Marlon Magnon Valdevino Leite \\ Robson Alves Bussolin \\ José Angelo Barela \\ Universidade Cruzeiro do Sul
}

\begin{abstract}
Resumo-O objetivo deste estudo foi verificar os efeitos de diferentes contextos no desenvolvimento das habilidades motoras fundamentais e no crescimento somático de crianças no ensino infantil. Participaram deste estudo 50 crianças: 25 tiveram educação física com um professor da área e 25 crianças tiveram atividades com o professor responsável pela turma. Crianças foram avaliadas no início e final do ano letivo quanto a dados antropométricos, dobras cutâneas, medidas de atividade física e realizaram os subtestes do TGMD-2. No início do ano letivo, nenhuma diferença foi observada entre os grupos. No final do ano letivo, crianças que tiveram atividades com o professor da sala apresentaram redução no nível de atividade física, enquanto que crianças com educação física com o professor especialista apresentaram manutenção no nível de atividade física e melhor desenvolvimento das habilidades motoras. O envolvimento em diferentes contextos de aulas de educação física não é suficiente para promover alteração somática em crianças ao longo do ano letivo. Entretanto, educação física com o professor especialista melhora o desenvolvimento das habilidades motoras fundamentais e promove manutenção do nível de atividade física em crianças no ensino infantil.
\end{abstract}

Palavras-chaves: desenvolvimento motor, habilidades motoras fundamentais, educação física

Abstract-“Motor development and somatic growth of children with different contexts in kindergarten.” The aim of this study was to investigate the effects of different contexts on fundamental motor skill development and on somatic growing of children in kindergarten. Fifty children participated in this study: 25 received physical education from a specialized teacher and 25 children received activities from a non-specialized teacher. Children were evaluated at the beginning and end of the school year, regarding anthropometric, skin fold, physical activity level, and TGMD-2. At the beginning of school year, no difference was observed between both groups in any measure. At the end of the year, children who received activities from a non-specialized teacher showed reduced level of physical activities whereas children who received physical education from a specialized teacher maintained the level and presented better fundamental motor skill development. Different contexts of physical education activities do not promote somatic differences along the school year. However, physical education from a specialized teacher improves fundamental motor skill development and maintenance of physical activity enrolment of children in kindergarten.

Keywords: motor development, fundamental motor skills, physical education

Resumen-“Desarrollo motriz y crecimiento somático de niños con diferentes contextos en la educación primaria.” El objetivo de este estudio fue comprobar los efectos de distintos contextos en el desarrollo de las capacidades motrices fundamentales y el crecimiento somático de niños en la educación primaria. Participaron de este estudio 50 niños, la mitad tuvo clases de educación física con un profesor del área de educación física y la otra mitad tuvo actividades con el profesor responsable del grupo. Se evaluaron al principio y final del curso escolar lo relativo a datos antropométricos, pliegues cutáneos y medidas de actividad física, además de realizar sub-tests del TGMD2. No hubo diferencia en las evaluaciones en el inicio del curso escolar. Al final del curso escolar el niños con actividades con el profesor del grupo presentó una reducción del nivel de actividad física, mientras niños con actividades con el profesor del educación física no presentó ninguna modificación y también un mejor desarrollo de las capacidades motrices. Conclusión: Participación en distintos contextos de la clase de educación física no es suficiente para promover una modificación somática en los niños, sin embargo, promueve una mejora en el desarrollo de las capacidades motrices fundamentales y manutención del nivel de actividad física.

Palabras claves: desarrollo motriz, capacidades motrices fundamentales, educación física 


\section{Introdução}

Os hábitos da vida moderna tendem causar alterações nas experiências e vivências motoras. De forma geral, temos observado uma redução drástica na necessidade de movimentos realizados no cotidiano, pelo menos aqueles considerados amplos, que têm sido substituídos por movimentos que envolvem grupos musculares menores. Essas alterações no repertório motor também têm sido observadas em crianças, com estas envolvidas cada vez mais cedo com aparelhos e jogos eletrônicos, em detrimento de realizar as atividades e brincadeiras tradicionais que envolvam ações motoras grossas, como por exemplo, as habilidades motoras fundamentais.

Outra constatação importante, nos dias atuais, é o aumento do índice de sobrepeso e obesidade, sendo que segundo o Instituto Brasileiro de Geografia e Estatística (IBGE, 2010), metade dos brasileiros está acima do peso. Essa constatação é, ainda, mais alarmante considerando que excesso de peso também tem sido observado em crianças, sendo que uma em cada três crianças de 5 a 9 anos apresenta excesso de peso (IBGE, 2010). Excesso de peso, na maioria dos casos, decorre de um desequilíbrio entre a ingestão calórica e o gasto energético, favorecendo o primeiro em relação ao segundo: consumo excessivo de nutrientes combinado com o crescente aumento do sedentarismo.

Considerando os efeitos deletérios da obesidade, a realização de movimentos que promovam gasto calórico, incluindo aqueles considerados habilidades motoras grossas (Gallahue \& Donnelly, 2008), deve ser estimulada e certamente a atuação do professor de educação física como promotor de atividades (Pellegrini \& Barela, 1998) assume papel de extrema importância. Tal sugestão decorre do fato de que, da mesma forma que diversas outras atividades, o prazer em realizar atividade física deve ser resultado de um processo construído ao longo de anos, tendo como uma das instâncias importantes a educação física escolar.

A aquisição e o refinamento de habilidades motoras são influenciados por muitos fatores (Thelen, 1995; Thelen \& Smith, 1994), sendo estes fatores denominados de restrições (Newell, 1986). Essa visão multicausal, com muitos fatores influenciando a aquisição e o refinamento das habilidades motoras, decorre do fato de que o ser em desenvolvimento é um sistema complexo, composto e influenciado por inúmeros fatores (Barela, 2001) e que mudanças desenvolvimentais decorrem de mudanças no conjunto destes fatores ou restrições (Clark, 1994). Embora o desenvolvimento motor pareça similar entre os seres humanos, diversas características são específicas ao ser em desenvolvimento. Essas especificidades no desenvolvimento motor são decorrentes de características individuais (orgânicas, psicológicas, motivacionais, etc.) e também do ambiente no qual o ser em desenvolvimento está inserido.

Como parte do ambiente, oportunidade de prática estruturada e instrução apropriada são fatores determinantes para que novas habilidades motoras sejam adquiridas e, principalmente, refinadas ao longo do ciclo desenvolvimen- tal, incluindo neste repertório as habilidades motoras fundamentais tais como correr, saltar, chutar, arremessar, receber, entre outras. A influência ambiental no processo desenvolvimental, tais como programas de atividade física e aulas de educação física, tem sido sugerida por diversos estudiosos (Clark, 2007; Gallahue \& Donnelly, 2008) e demonstrada empiricamente (Braga, Krebs, Valentini, \& Tkac, 2009; Cotrim, Lemos, Néri Jr., \& Barela, 2011; Lemos, Avigo, \& Barela, 2012).

Prática estruturada ou organizada é a atividade propiciada às crianças e adolescentes com objetivos compatíveis com o nível desenvolvimental esperado para a respectiva idade das crianças e adolescentes. As atividades propiciadas pelo professor devem ser organizadas de forma a garantir que habilidades motoras sejam adquiridas e refinadas de acordo com o esperado para a idade da criança. Portanto, atividades organizadas pelo professor devem ser estruturadas para garantir que o desenvolvimento pleno seja alcançado, considerando as necessidades e competências motoras esperadas nos respectivos períodos desenvolvimentais (Clark, 2007; Gallahue \& Donnelly, 2008).

Ao longo de muito tempo foi assumido que prática organizada e instrução apropriada, propiciadas por aulas de educação física para a aquisição de habilidades motoras, deveriam ocorrer apenas a partir dos 10 anos de idade. Por volta dessa idade deveria ocorrer a introdução e a aprendizagem de habilidades motoras específicas às modalidades desportivas mais proeminentes no ambiente (SEE, 2008).

A proposta de educação física apenas a partir da primeira década de vida decorreu, principalmente, do entendimento de que as habilidades motoras fundamentais desenvolvidas antes dos 10 anos de idade eram adquiridas naturalmente, não necessitando, portanto, de prática estrutura e instrução propiciadas por uma situação de aprendizagem formal. Entretanto, a visão de que crianças adquirem as habilidades motoras fundamentais naturalmente (e.g., Gallahue, 1982; Gallahue \& Donnelly, 2008) e, portanto, não necessitam de atuação de um profissional e prática estruturada não corresponde com a realidade. Estudos recentes têm indicado que aulas de educação física contribuem decisivamente para o refinamento das habilidades motoras fundamentais (Cotrim et al., 2011; Lemos et al., 2012; Pang \& Fong, 2009) e, portanto, questionam a importância de aulas de educação física apenas após a primeira década de vida.

A falta de desenvolvimento pleno das potencialidades motoras e, em específico das habilidades motoras fundamentais, tem sido demonstrada no conjunto de habilidades motoras em geral (e.g., Braga et al., 2009). A falta de oportunidade de prática sistematizada e estruturada com objetivos de proporcionar experiências motoras diversificadas, e a falta de instruções apropriadas têm sido indicadas como razões para que crianças não alcancem níveis mais elevados de desempenho motor nas habilidades motoras fundamentais (Cotrim et al., 2011; Lemos et al., 2012; Pang \& Fong, 2009), ficando aquém do nível esperado para as respectivas idades (e.g., Braga et al., 2009; Ferraz, 1992; Valentini, 2002). 
Por outro lado, programas de intervenção motora (Braga et al., 2009) e participação em aulas de educação física (Cotrim et al., 2011; Lemos et al., 2012) têm indicado que crianças podem exibir melhora na execução de habilidades motoras fundamentais. Estes resultados são muito importantes e promissores para aqueles que atuam na área de educação física. Primeiro, tais resultados indicam que intervenção estruturada provoca alterações no desenvolvimento motor mesmo de crianças jovens. Segundo, indicam e corroboram a importância das aulas de educação física para o desenvolvimento daquelas habilidades motoras consideradas fundamentais, mesmo no ensino infantil e fundamental.

A pergunta que surge, entretanto, é se crianças com atividade estrutura para promover a proficiência motora de habilidades motoras fundamentais também apresentariam maior engajamento em atividade física de forma geral e, se assim ocorrer, esse maior envolvimento promoveria alterações somáticas que poderiam prevenir ou amenizar os problemas relacionados ao sobrepeso e obesidade. Tal questão decorre do fato de que, como em outros domínios, o envolvimento em atividades motoras perpassa pela competência em realizar a atividade proposta. Assim, crianças que apresentam dificuldades em realizar os movimentos envolvidos na atividade, tendem a se afastar do envolvimento da mesma. No domínio motor, essa dificuldade foi denominada de barreira de proficiência (Clark, 2007; Gallahue \& Donnelly, 2008; Seefeldt \& Haubenstricker, 1982). O objetivo do presente estudo foi verificar os efeitos de diferentes contextos de aulas ministradas no desenvolvimento das habilidades motoras fundamentais e no crescimento somático de crianças no ensino infantil.

\section{Método}

\section{Participantes}

A amostra foi inicialmente composta por 50 crianças, de ambos os gêneros, com idades entre 4 e 6 anos, regularmente matriculadas no ensino infantil de uma escola municipal da cidade de Guarulhos. Vinte e cinco crianças foram selecionadas para, ao longo do ano letivo, receber aulas de educação física duas vezes por semana com um professor especializado da área. Essas crianças formaram o grupo denominado com professor de educação física (PEF). Vinte e cinco crianças foram selecionadas para terem atividades motoras com o professor responsável pela turma, e formaram o grupo com professor de sala de aula (PSA). Os procedimentos do presente estudo foram aprovados pelo comitê de ética institucional e os responsáveis pelas crianças assinaram um Termo de Consentimento Livre e Esclarecido, autorizando a participação das mesmas no estudo.

As crianças do grupo PSA tiveram aulas de atividade motora ministradas pelo professor polivalente, responsável pela turma, aulas essas que predominantemente foram constituídas de atividades recreativas, realizadas em um playground instalado na escola. Diferentemente, as crian- ças do grupo PEF tiveram, ao longo do ano letivo do ensino infantil, aulas de educação física ministradas por profissional da área. As atividades referentes às aulas de educação física seguiram o conteúdo sugerido pelo sistema educacional, dentro da realidade profissional e de estrutura da escola.

\section{Procedimentos}

As crianças foram avaliadas em dois momentos, denominados de pré- e pós-teste. A coleta de dados do préteste ocorreu no início do ano durante o mês de março (primeiro mês letivo), e a coleta pós-teste ocorreu no final do mesmo ano letivo no mês de novembro (último mês letivo), perfazendo um intervalo de aproximadamente oito meses entre o pré- e o pós-teste.

Após a obtenção das informações iniciais, as medidas de massa corporal e estatura foram obtidas, utilizando uma balança digital (Ironma Tanika BC-554) e um estadiômetro (estadiômtro compacto tipo trena, Sanny). Ainda, neste momento, foram obtidas medidas de dobras cutâneas de três pontos específicos: tricipital; abdominal e panturrilha média. A obtenção destas medidas seguiu o protocolo de aquisição das mesmas, sendo realizadas três medidas do lado direito para cada ponto, utilizando um plicômetro digital (Prime Vision DGI). Os mesmos procedimentos foram repetidos no último mês de coleta de dados (mês de novembro).

Ao longo do primeiro mês de realização do projeto (mês de março), medidas de atividade física foram obtidas das crianças. Neste caso, pedômetros (pedômetro Tech Line) foram afixados na vestimenta das crianças, altura do quadril, antes do início das aulas de educação física (PEF) e das atividades com a professora da turma (PSA). Os pedômetros foram afixados em dez crianças, em cada dia, definidas de forma aleatória, sendo que no primeiro mês, pelo menos três medidas de atividade física foram obtidas para cada criança. O mesmo ocorreu no último mês de coleta de dados (mês de novembro).

Posteriormente, as crianças foram filmadas, em espaço da própria escola, realizando as habilidades motoras dos subtestes locomotor e controle de objeto do Teste de Desenvolvimento Motor Grosso, segunda edição, TGMD-2 (Test of Gross Motor Development, 2nd edition), como realizado em oportunidades anteriores (Cotrim et al., 2011; Lemos et al., 2012). Para tanto, duas câmeras digitais (Sony, modelo DCR-HC96) foram posicionadas de tal forma que uma câmera possibilitou a filmagem das habilidades do subteste locomotor e a outra câmera possibilitou a filmagem das habilidades do subteste controle de objeto. A identidade das crianças foi preservada, sendo que cada criança foi identificada por meio de numeração definida anteriormente às filmagens.

Antes da realização da filmagem das habilidades requeridas no TGMD-2, o instrutor forneceu instrução verbal e realizou uma demonstração sobre a habilidade a ser realizada, seguindo as instruções para aplicação do TGMD2 (Ulrich, 2000). Após a instrução e demonstração, a criança realizou a habilidade solicitada para garantir que ela tivesse 
entendido a tarefa a ser realizada. Caso a criança não entendesse ou conseguisse realizar a habilidade motora solicitada, nova instrução, demonstração e prática eram permitidas. Cada criança realizou pelo menos três repetições de cada habilidade motora do TGMD-2, sendo uma para prática e duas tentativas válidas para posterior análise.

As instruções foram dadas às crianças por dois profissionais de educação física treinados na aplicação do TGMD-2, sendo que um ficou responsável pela aplicação do subteste locomotor e o outro do subteste controle de objeto, tanto para o pré- quanto para o pós-teste. A realização dos procedimentos dos dois subtestes, por cada criança, durou por volta de 15 a 20 minutos.

\section{Análise dos dados}

Após a obtenção dos dados, os mesmos foram organizados de acordo com cada turma e teste (pré- e pós-) que os mesmos foram obtidos. Com relação à análise das habilidades motoras fundamentais, os valores para os critérios de desempenho para cada habilidade motora foram obtidos. Para tanto, as imagens referentes à performance das habilidades motoras de cada subteste do TGMD-2 foram inspecionadas por três avaliadores, devidamente treinados, utilizando um reprodutor de DVD e uma TV (LCD32-Sony). Estes avaliadores analisaram as duas tentativas para cada habilidade, indicando o valor correspondente à realização dos movimentos com base nos critérios de desempenho indicados no teste (Ulrich, 2000), em formulário de análise específica.

Com base na análise das habilidades motoras, considerando os critérios de desempenho, os valores brutos para cada subteste foram calculados, podendo alcançar o máximo de 48 pontos. Neste caso, quanto mais próximo do valor máximo, melhor é o nível de desenvolvimento das crianças na realização das habilidades motoras fundamentais. As médias das dobras cutâneas foram obtidas, entre as três medidas realizadas, e um valor para cada localização da dobra foi alcançado. No primeiro e último mês de realização do projeto, a quantificação da atividade física das crianças foi mensurada obtendo a média dos passos realizados (passos), distância estimada percorrida com o pedômetro (Km) e a estimativa de dispêndio calórico (Kcal), durante o período que a criança permaneceu com os pedômetros. Estes procedimentos foram realizados no pré- e pós-teste.

\section{Análise estatística}

Três análises de variância (ANOVAs) e seis análises de multivariância (MANOVAs ) foram utilizadas. Uma ANOVA, tendo como fator grupo, foi utilizada para comparar a idade cronológica entre as crianças dos grupos PEF e PSA. Duas MANOVAs, tendo como fator grupo, foram utilizadas para comparar as medidas antropométricas (peso, estatura e IMC) entre as crianças dos grupos PEF e PSA, na realização do pré-teste e na realização do pós-teste. Duas MANOVAs, tendo como fator grupo, foram utilizadas para comparar as medidas de dobras cutâneas (triciptal, abdominal e panturrilha média) entre as crianças dos grupos PEF e PSA, na realização do pré-teste e na realização do pós-teste. Duas ANOVAs, tendo com fatores grupo e teste, foram utilizadas para comparar os valores brutos do TGMD-2 entre as crianças dos grupos PEF e PSA no pré- e pós-teste, este último tratado como medida repetida, sendo uma análise envolvendo os valores brutos do subteste locomotor e a outra análise os valores brutos do subteste controle de objeto. Finalmente, duas MANOVAs, tendo como fator grupo, foram utilizadas para comparar as medidas de atividade física (número de passos, distância percorrida e dispêndio calórico) entre as crianças dos grupos PEF e PSA, na realização do pré-teste e na realização do pós-teste. Quando necessário, testes univariados foram realizados. Todas as análises foram realizadas utilizando o SPSS (SPSS for Windows, versão 10) e o nível de significância foi mantido em 0,05.

\section{Resultados}

\section{Dados antropométricos}

A Tabela 1 apresenta informação sobre os dados antropométricos das crianças analisadas nos momentos de realização do pré e pós-teste. ANOVA não revelou qualquer diferença $(p>0,05)$ para idade cronológica, entre os dois grupos. Da mesma forma, MANOVA não revelou qualquer diferença $(p>0,05)$ para as variáveis, peso, estatura e IMC, entre os grupos.

\section{Dobras cutâneas}

A Tabela 2 apresenta informação sobre as dobras cutâneas das crianças nos momentos de realização do pré- e

Tabela 1. Média e desvio-padrão da idade cronológica, peso corporal, estatura e índice de massa corporal (IMC) para as crianças sem aulas de educação física (SEF) e com aulas de educação física (CEF), no pré- e pós-teste.

\begin{tabular}{lllll}
\hline Crianças & Idade (anos) & $\begin{array}{l}\text { Peso corporal }(\mathrm{Kg}) \\
\text { pré / pós }\end{array}$ & $\begin{array}{l}\text { estatura }(\mathrm{m}) \\
\text { pré / pós }\end{array}$ & $\begin{array}{l}\text { IMC }\left(\mathrm{kg} / \mathrm{m}^{2}\right) \\
\text { pré / pós }\end{array}$ \\
\hline SEF & $4,4(0,28)$ & $21,0(7,20) / 22,2(6,83)$ & $1,09(0,06) / 1,18(0,07)$ & $17,1(4,0) / 15,4(3,2)$ \\
CEF & $4,5(0,25)$ & $20,1(3,48) / 22,3(5,43)$ & $1,10(0,03) / 1,21(0,04)$ & $16,3(2,5) / 15,2(3,3)$ \\
\hline
\end{tabular}


pós-teste. MANOVA não revelou qualquer diferença ( $p>$ $0,05)$ para as três medidas de dobras cutâneas entre as crianças dos grupos CEF e SEF, tanto no pré- quanto no pós-teste.

\section{Desenvolvimento das habilidades motoras fundamentais}

Figura 1 apresenta os valores brutos do subteste locomotor do TGMD-2 para ambos os grupos de crianças. ANOVA revelou diferença entre o pré- e o pós-teste, $\mathrm{F}_{1,36}$ $=38,02 ; p<0,001$, e interação grupo e teste, $\mathrm{F}_{1,36}=5,62 ; p<$ 0,05 . Nesse caso, observa-se que ambos os grupos aumentaram os valores brutos, indicando melhora na realização das habilidades do subteste locomotor, porém o aumento observado para o grupo PEF foi maior que o observado para o grupo PSA.

Figura 2 apresenta os valores brutos do subteste controle de objeto do TGMD-2 para ambos os grupos de crianças. ANOVA revelou diferença para teste, $\mathrm{F}_{1,35}=25,97$; $p<0,001$, e marginalidade para a interação grupo e teste, $\mathrm{F}_{1,35}=3,35 ; p$ $=0,07$. Da mesma forma que observado para as habilidades do subteste locomotor, os valores do escore bruto, para o subteste controle de objeto, aumentou do pré para o pósteste, sendo que o aumento observado para o grupo PEF foi marginalmente maior que o aumento observado para o grupo PSA.

\section{Quantificação da atividade física}

A Tabela 3 apresenta informação sobre o número de

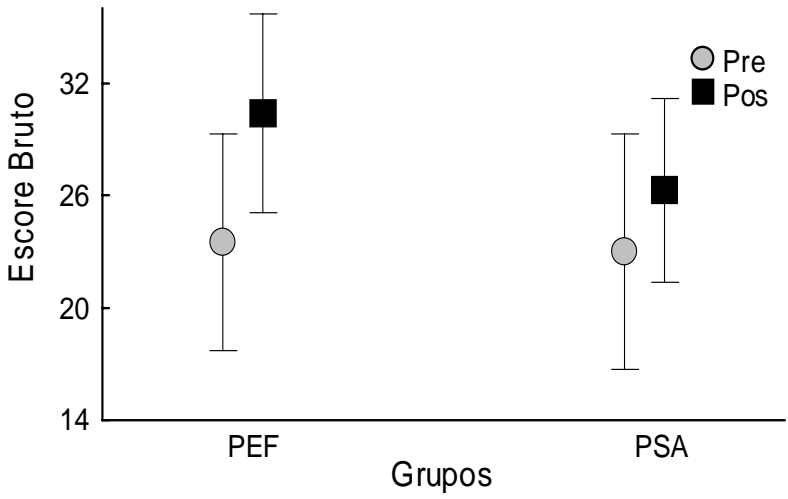

Figura 1. Média e desvio-padrão dos valores brutos para o subteste locomotor das crianças sem educação física (SEF) e com educação física (CEF), no pré- e pós-teste.

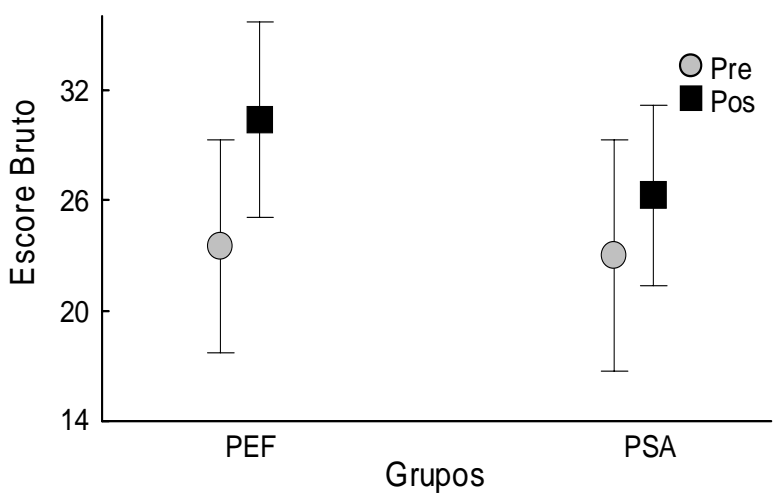

Figura 2. Média e desvio-padrão dos valores brutos do subteste controle de objeto das crianças sem educação física (SEF) e com educação física (CEF), no pré- e pós-teste.

Tabela 2. Média e desvio-padrão das medidas de dobras cutâneas triciptal, abdominal e panturrilha média para as crianças sem aulas de educação física (SEF) e com aulas de educação física (CEF), nos pré- e pós-teste.

\begin{tabular}{llll}
\hline Crianças & $\begin{array}{l}\text { Triciptal }(\mathrm{mm}) \\
\text { pré / pós }\end{array}$ & $\begin{array}{l}\text { Abdominal }(\mathrm{mm}) \\
\text { pré / pós }\end{array}$ & $\begin{array}{l}\text { Panturrilha média }(\mathrm{mm}) \\
\text { pré / pós }\end{array}$ \\
\hline SEF & $10,6(3,2) / 11,2(4,8)$ & $9,9(5,3) / 10,4(6,2)$ & $11,5(3,0) / 12,6(5,7)$ \\
CEF & $10,0(2,8) / 11,5(3,1)$ & $10,1(3,4) / 10,9(5,3)$ & $11,5(4,5) / 11,8(4,3)$
\end{tabular}

Tabela 3. Média e desvio-padrão do número de passos, estimativa da distância percorrida e do dispêndio calórico para as crianças sem aulas de educação física (SEF) e com aulas de educação física (CEF), no pré e pós-teste.

\begin{tabular}{llll}
\hline Crianças & $\begin{array}{l}\mathrm{N}^{0} \text { passos (passos) } \\
\text { pré / pós }\end{array}$ & $\begin{array}{l}\text { Distância }(\mathrm{Km}) \\
\text { pré / pós }\end{array}$ & $\begin{array}{l}\text { Calorias (cal) } \\
\text { pré / pós }\end{array}$ \\
\hline SEF & $1256(629) / 826(490)$ & $0,70(0,21) / 0,52(0,26)$ & $12,7(7,6) / 6,5(4,0)$ \\
CEF & $1193(382) / 1193(444)$ & $0,78(0,39) / 0,73(0,22)$ & $14,0(5,3) / 11,5(4,0)$
\end{tabular}


passos, estimativa da distância percorrida e do dispêndio calórico das crianças analisadas nos momentos de realização do pré e pós-teste. MANOVA não revelou qualquer diferença $(p>0,05)$ para as três medidas entre os grupos PEF e PSA, no pré-teste. Já no pós-teste, MANOVA revelou diferença entre os grupos PEF e PSA, Wilks' Lambda $=0,554, \mathrm{~F}_{3,32}=$ 8,57; $p<0,001$. Análises univariadas indicaram que a diferença entre os grupos ocorreu para todas as variáveis, sendo que o grupo PEF realizou mais passos, $\mathrm{F}_{1,34}=5,47 ; p<0,05$; percorreu distância maior, $\mathrm{F}_{1,34}=6,11 ; p<0,05$, e teve dispêndio maior de calorias, $\mathrm{F}_{1,34}=13,76 ; p<0,005$, do que o grupo PSA. Na realidade, dos dados apresentados na Tabela 3 observa-se que no último mês de observação as crianças do grupo PEF mantiveram o mesmo nível de atividade física observado no primeiro mês de observação, enquanto que as crianças do grupo PSA apresentaram diminuição do nível de atividade física.

\section{Discussão}

O objetivo do presente estudo foi verificar os efeitos de diferentes contextos de aulas ministradas no desenvolvimento das habilidades motoras fundamentais e no crescimento somático de crianças no ensino infantil. Os resultados mostraram diferenças claras no desenvolvimento das habilidades motoras grossas, com desempenho superior de crianças que tiveram aulas de educação física com professor da área comparado com o desenvolvimento das crianças que tiveram atividade com uma professora polivalente. Estes resultados corroboram resultados de estudos anteriores (e.g., Lemos et al., 2012) confirmando que aulas ministradas por profissional da área influenciam o desenvolvimento das habilidades motoras fundamentais, mesmo em crianças do ensino infantil. Os resultados também indicam que embora a atividade no ensino infantil não produza alteração nas medidas antropométricas e alteração no nível de composição corporal, crianças com atividade ministrada por especialista da área apresentam maior movimentação, com nível mais elevado de atividade física. Essa constatação é importante e pode constituir fator crucial para que crianças engajem em atividades, evitando assim redução no envolvimento em situações que demandem atividade física.

Estudos anteriores demonstraram a importância e os efeitos benéficos para a melhora na realização de habilidades motoras fundamentais de crianças envolvidas em programas específicos de intervenção (Akbari et al., 2009; Bakhtiari, Shafinia, \& Ziaee, 2011; Braga et al., 2009; Brauner \& Valentini, 2009) ou em aulas regulares de educação física (Cotrim et al., 2011; Lemos et al., 2012). Os resultados do presente estudo corroboram os efeitos positivos da atuação do profissional de educação física para a promoção do desenvolvimento das habilidades motoras fundamentais. Tal constatação é importante por diversos motivos. Primeiro, se oportunidade de prática e instrução apropriada ocorrerem em momento apropriado, considerando os períodos mais sensíveis para a aquisição e refinamento das habilidades motoras que é por volta dos 7 e 8 anos para as habilidades motoras fundamentais (Clark, 2007; Gallahue \& Donnelly, 2008), a partir de aulas de educação física, o desenvolvimento das habilidades seria alcançado em patamares mais elevados. Segundo, acontecendo desenvolvimento motor mais avançado, não haveria necessidade de crianças engajarem em programas especiais de intervenção motora, pois as aulas de educação física já propiciaram os estímulos e condições necessárias para o desenvolvimento motor esperado e desejado.

Ainda, considerando que a dificuldade em alcançar execuções mais elaboradas das habilidades motoras fundamentais, pode ser uma dificuldade e até um fator impeditivo para o engajamento e a participação em atividades motoras, com objetivos variados (brincadeiras, danças, recreação, esportes, etc.), os resultados observados no presente estudo assumem também uma importância adicional. A dificuldade na execução de habilidades motoras fundamentais, comum para algumas das crianças, conforme observado em estudos que demonstraram atraso na execução das habilidades motoras fundamentais (Bonifacci, 2004; Braga et al., 2009; Brauner \& Valentini, 2009; Castro, 2008; Lopes, Lopes, \& Pereira, 2006; Pick, 2004) pode contribuir de alguma forma para prejudicar ou impedir o avanço no curso desenvolvimental dessas crianças. Estudos recentes têm demonstrado uma associação positiva entre o nível de proficiência motora e o envolvimento em atividade física (Wrotniak, Epstein, Dorn, Jones, \& Rondilis, 2010) e que ocorre uma associação entre desenvolvimento motor, mesmo em idades jovens, com a prática e envolvimento esportivo na adolescência (Ridgway et al., 2009). Assim, nível baixo de desenvolvimento motor, causando dificuldades na execução das habilidades motoras fundamentais, constitui uma barreira para o desenvolvimento motor pleno das crianças, a barreira de proficiência motora, conforme apontado por alguns autores (Clark, 2007; Gallahue, 1989; Seefeldt \& Haubenstricker, 1982). Portanto, aulas de educação física com profissional da área, mesmo em idades mais tenras como de crianças matriculadas no ensino infantil, promovem mudanças desenvolvimentais importantes na execução de habilidades motoras fundamentais.

Com base nos resultados do presente estudo, podemos sugerir que condições apropriadas para a promoção de desenvolvimento, mesmo no ensino infantil, perpassam pela atuação do professor de educação física. Neste caso, o professor tem papel crucial atuando como promotor de atividades estruturadas e planejadas, de acordo com a faixa etária, e atuando como fornecedor de informação, corrigindo e orientando a realização correta de habilidades motoras, mesmo das consideradas fundamentais, como anteriormente sugerido (Pellegrini \& Barela, 1998). Mais importante, ainda, é a observação que atividade estrutura e ministrada por um profissional da área mantém o interesse no envolvimento de atividade física. Crianças que tiveram aulas com profissional da área mantiveram o mesmo nível de atividade física. Considerando que envolvimento em atividades pode ser importante para a satisfação do executante (Morano, Colella, 
\& Capranica, 2011) e que obesidade está associada com atrasos no desenvolvimento de habilidades motoras em idades jovens (Slining, Adair, Goldman, Borja, \& Bentley, 2010), a manutenção e a promoção do envolvimento em atividades físicas são de extrema importância. Nenhuma alteração na composição corporal foi observada ao longo de um ano letivo (8 meses). Possivelmente este período de tempo seja muito curto ou que as crianças sejam muito jovens para que tais alterações venham a ocorrer em tal período de tempo. Entretanto, essas suposições necessitam ser melhor investigadas.

Os resultados do presente estudo também são importantes para evidenciar a influência multicausal no desenvolvimento motor. Como sugerido, desenvolvimento motor é um processo complexo, resultante das oportunidades que a criança vivencia ao longo do processo desenvolvimental (Barela, 1999; Clark, 1994; Thelen, 1995). Apesar da influência de muitos fatores e aspectos, alguns podem ter importância capital no delineamento do curso desenvolvimental. No presente estudo, crianças que tiveram educação física com um professor de educação física apresentaram maiores avanços no desenvolvimento motor. Portanto, o papel do professor funcionou como um fator crucial para promoção do desenvolvimento.

Como mencionado anteriormente, o entendimento de que o desenvolvimento de habilidades motoras fundamentais ocorre de forma natural, como transpareceu em algumas propostas (Gallahue, 1982), governado por princípios neuromaturacionais (p. ex., Gesell, 1933), tem sido apontado como um enorme engano que permeou e ainda permeia as propostas de educação física (Clark, 2007). Os resultados do presente estudo fornecem suporte empírico da importância de oportunidade e prática estruturada e sistematizada para que, as habilidades motoras fundamentais sejam desenvolvidas em sua plenitude. Mais ainda, os resultados sugerem que habilidades motoras fundamentais são adquiridas naturalmente, conforme a criança explora as suas potencialidades motoras, com sugerido em diversos modelos desenvolvimentais (Gallahue \& Donnelly, 2008). Entretanto essas habilidades não são refinadas naturalmente. Crianças, ao longo da primeira década de vida, têm potencial para realizar as habilidades motoras fundamentais com desenvoltura e adaptável ao contexto. Porém para que tais potencialidades se materializem, crianças necessitam de estímulos ambientais na forma de instrução e prática apropriadas para que esse potencial seja plenamente revertido em ações motoras mais refinadas.

O presente estudo apresenta algumas limitações que necessitam ser apontados. Primeiro, o número de crianças participantes do estudo pode ser considerado pequeno. Segundo, a atuação de cada professor, especialista e polivalente, não foi detalhadamente controlada, o mesmo ocorrendo o conteúdo desenvolvido pelos mesmos. Terceiro, a obtenção de informação sobre o nível de atividade física é muito complicada, pois a simples colocação de um aparelho (no caso o pedômetro) pode provocar mudanças no hábito e no nível de atividade, principalmente, se tratando de crianças com idade entre 4, 5 e 6 anos. Assim, os dados de atividade física, do presente estudo, devem ser interpretados com cautela, pois mesmo tomando todos os cuidados, algumas alterações podem ter ocorrido. Finalmente, seria interessante acompanhar as crianças ao longo de um período para verificar possíveis efeitos nível de desenvolvimento motor, da composição corporal e no envolvimento em atividade física.

\section{Referências}

Akbari, H., Abdoli, B., Shafizadeh, M., Khalaji, H., Hajihosseini, S., \& Ziaee, V. (2009). The effect of traditional games in fundamental motor skill development in 7-9 year-old boys. Iranian Journal of Pediatrics, 19(2), 123-129.

Bakhtiari, S., Shafinia, P., \& Ziaee, V. (2011). Effects of selected exercises on elementary school third grade girl students' motor development. Asian Journal of Sports Medicine, 2(1), 51-56.

Barela, J.A. (1999). Aquisição de habilidades motoras: do inexperiente ao habilidoso. Motriz, 5(1), 53-57.

Barela, J.A. (2001). Ciclo percepção-ação no desenvolvimento motor. In L. A. Teixeira (Ed.), Avanços em Comportamento Motor (pp. 41-61). Rio Claro: Movimento.

Bonifacci, P. (2004). Children with low motor ability have lower visual-motor integration ability but unaffected perceptual skills. Human Movement Science, 23, 157-168.

Braga, R.K., Krebs, R.J., Valentini, N.C., \& Tkac, C.M. (2009). A influência de um programa de intervenção motora no desempenho das habilidades locomotoras de crianças com idade entre 6 e 7 anos. Revista da Educação Física/UEM, 20(2), 171-181.

Brauner, L.M., \& Valentini, N.C. (2009). Análise do desempenho motor de crianças participantes de um programa de atividades físicas. Revista da Educação Física/UEM, 20(2), 205-216.

Castro, M.B. (2008). A influência do contexto nas habilidades motoras fundamentais de pré escolares e escolares. Dissertação de Mestrado. Escola de Educação Física, Universidade Federal do Rio Grande do Sul, Rio Grande do Sul.

Clark, J.E. (2007). On the problem of motor skill development. JOPERD, 78(5), 39-45.

Clark, Jane E. (1994). Motor Development. Encyclopedia of Human Behavior, 3, 245-255.

Cotrim, J.R., Lemos, A.G., Néri Jr., J.E., \& Barela, J.A. (2011). Desenvolvimento de habilidades motoras fundamentais em crianças com diferentes contextos escolares. Revista de Educação Física/UEM, 22(4), 523-533.

Ferraz, O.L. (1992). Desenvolvimento do padrão fundamental de movimento correr em crianças: um estudo semi-longitudinal. Revista Paulista de Educação Física, 6(1), 26-34.

Gallahue, D.L. (1982). Understanding Motor Development in Children. Boston, MA: John Wiley \& Sons, Inc.

Gallahue, D.L. (1989). Motor Developmentalists - All? Motor Development Academy Newsletter - National Association of Sport and Physical Education, 10(1), 1-5.

Gallahue, D.L., \& Donnelly, F.C. (2008). Educação Física Desenvolvimentista para Todas as Crianças (4a ed.). São Paulo, SP: Phorte.

Gesell, A. (1933). Maturing and the patterning of behavior. In C. Murchison (Ed.), A handbook of child psychology (2nd ed., pp. 209-235). New York: Russell \& Russell.

IBGE. (2010). Antropometria e estado nutricional de crianças, adolescentes e adultos no Brasil. Retrieved 29/03/2013, 2013

Lemos, A.G., Avigo, E.L., \& Barela, J.A. (2012). Physical Education 
in kindergarten promotes fundamental motor skill development. Advances in Physical Education, 2(1), 17-21.

Lopes, Luis, Lopes, Vitor Pires, \& Pereira, Beatriz. (2006). Atividade Física no recreio escolar: Estudo de intervenção em crianças dos seis aos doze anos. Revista Brasileira de Educação Física e Esporte, 20(4), 271-280.

Morano, M., Colella, D., \& Capranica, L. (2011). Body image, perceived and actual physical abilities in normal-weight and overweight boy involved in individual and team sports. Journal of Sports Sciences, 29(4), 355-362.

Newell, K.M. (1986). Constraints on the development of coordination. In M. G. Wade \& H. T. A. Whiting (Eds.), Motor development in children: Aspects of coordination and control (pp. 341-360). Boston, MA: Martin Nighoff.

Pang, A.W.-Y., \& Fong, D. T.-P. (2009). Fundamental motor skill proficiency of Hong Kong children aged 6-9 years. Research in Sports Medicine, 17, 125-144.

Pellegrini, A.M., \& Barela, J.A. (1998). O que o professor deve saber sobre o desenvolvimento motor de seus alunos. In M. C. d. O. Micotti (Ed.), Alfabetização: Assunto para pais e mestres (pp. 69-80). Rio Claro: Instituto de Biociências.

Pick, R.K. (2004). A influência de um programa de intervenção motora inclusiva no desenvolvimento motor e social da criança com atrasos motores. Escola de Educação Física, Universidade Federal do Rio Grande do Sul, Rio Grande do Sul.

Ridgway, C. L., Ong, K.K., Tammelin, T.H., Sharp, S., Ekelund, U., \& Jarvelin, M.-R. (2009). Infant motor development predicts sports participation at age 14 years: Northern Finland birth cohort of 1966. PLoS One, 4(8), e6837.

Proposta Curricular do Estado de São Paulo: Educação Física (2008).

Seefeldt, V., \& Haubenstricker, J. (1982). Patterns, phase, or stages: An analytical model for the study of developmental movement. In J. A. S. Kelso \& J. E. Clark (Eds.), The development of movement control and coordination (pp. 309-318). New York, NY: John Wiley \& Sons, Ltd.

Slining, M., Adair, L.S., Goldman, B.D., Borja, J.B., \& Bentley, M. (2010). Infant overweight is associated with delayed motor development. The Journal of Pediatrics, 157(1), 20-25.e21.

Thelen, E. (1995). Motor development: A new synthesis. American Psychologist, 50(2), 79-95.

Thelen, E., \& Smith, L.B. (1994). A dynamic systems approach to the development of cognition and action. Cambridge: MIT Press.

Ulrich, D.A. (2000). Test of gross motor development-2 (2nd ed.). Austin, TX: PRO-ED.

Valentini, N.C. (2002). Percepções de competência e desenvolvimento motor de meninos e meninas: um estudo transversal. Movimento, 8(2), 51-62.

Wrotniak, B.H., Epstein, L.H., Dorn, Joan M., Jones, K., \& Rondilis, V.A. (2010). The relationship between motor proficiency and physical activity in children. Pedriatics, 118(6), e1758-e1765.

\section{Nota dos autores}

Décio Rodrigues e Eric Leal Avigo são mestrandos em Ciências do Movimento Humano, Universidade Cruzeiro do Sul.

Marlon Magnon Valdevino Leite e Robson Alves Bussolin são graduandos em educação física, Instituto de Ciências da Atividade Fisica e Esporte, Universidade Cruzeiro do Sul.

José Angelo Barela é docente na Universidade Cruzeiro do Sul.

\section{Correspondência}

José Angelo Barela

Universidade Cruzeiro do Sul, campus Liberdade

São Paulo, SP

Email: jbarela@rc.unesp.br

Artigo completo de trabalho apresentado no VIII Congresso Internacional de Educação Física e Motricidade Humana e XIV Simpósio Paulista de Educação Física.

Declaração de Conflito de Interesses: Os autores declararam ausência de conflitos de interesse no que diz respeito à pesquisa, autoria e/ou publicação deste artigo.

Manuscrito recebido em 18 de março de 2013

Manuscrito aceito em 3 de maio de 2013 